sionellen Ebene getroffen werden" (38) und weil sie darauf hinweist, dass „Veränderungen im Organisationsverhalten weniger über die Anpassung der offiziellen Organisationsziele legitimiert werden“ als über die „... operative Transformation allgemeiner Oberziele von besonderer Relevanz" (ebd.). Aus diesen Feststellungen gewinnt er später (Kap. 5 und 6) Anregungen für die Auswahl der zu untersuchenden Entscheidungen. Den Organisationsforscher macht indes stutzig, dass viele der dargestellten Aspekte nur für den öffentlich-rechtlichen Rundfunk Gültigkeit haben sollen: Das „PrinzipalAgent"-Problem ist nicht nur für öffentliche Unternehmen, sondern auch für privatwirtschaftliche Organisationen von erheblicher Relevanz, wie die Ereignisse um die Aktienblase in den 90er Jahren des 20. Jahrhunderts und die damit einhergehenden Bereicherungsorgien des oberen Managements überdeutlich belegen. Zielkonflikte gibt es auch bei privaten Rundfunkorganisationen, die ebenfalls mit kontingenten, sprich: „unklaren Kausalbeziehungen“ (S. 73) zu tun haben, die ein weites Terrain semantischer Beliebigkeit eröffnen. Zumindest aus organisationstheoretischer Warte ist die Situation zwischen privatwirtschaftlichen und öffentlich-rechtlichen Organisationen so verschieden nicht: Strategieentwicklung muss in jedem Fall intern geleistet werden und Entscheidungen erfahren hier wie dort meist eine ex-post Rationalisierung, eine wettbewerbsorientierte Programmierung ist hier wie dort schwierig, wiewohl sich idealtypische Programmierungen für die verschiedenen Anstaltstypen aufzeigen lassen (S. 215). Hier wie dort erweist sich „Expertise“ in der Programmplanung lediglich als eine auf Erfolg beruhende Heuristik, die sich aus einer Kombination von „science, art \& intuition“ ableitet, die aber dazu beiträgt, die Machtansprüche des TV-Managements zu legitimieren. Letzteres konstatiert Meier durchaus scharfsinnig. Die Konsequenzen einer organisationsorientierten Analyse werden allerdings nicht so deutlich herausgearbeitet, obwohl der Autor mit den einschlägigen Theorien vertraut zu sein scheint. Da hätte man mehr herausholen können. Auch die Beobachtung, dass sich in den letzten Jahren die Stimmen mehren, die einer privatwirtschaftlichen Organisation ehemals öffentlicher Aufgaben das Wort reden, ist allein für sich genommen keine hinreichende Begründung für das seit Jahren ziemlich unreflektiert propagierte Pri- vatisierungsmantra. Auf den Ergebnissen einer empirischen Strategieforschung kann es jedenfalls nicht aufbauen, da diese bereits Schwierigkeiten hat, ihre strategischen Erfolgsrezepte konzis darzustellen (S. 36).

Die kritische Überprüfung dieses Mantras ist indessen nicht das Thema der vorliegenden Studie, die insofern einzigartigen Charakter hat, als selten Material zur Verfügung gestellt wird, auf dessen Basis sich Entscheidungsprozesse in Organisationen nachvollziehen lassen. Mit welchen Anforderungen eine derartige Aufgabe verbunden ist und mit welchen Schwierigkeiten Wissenschaftler dabei zu kämpfen haben, lässt sich bei Meier detailliert verfolgen. Unter Berücksichtigung der vielen potenziellen Lesarten dieses quantitativ umfangreichen Werks (Methode, Rundfunkpolitik, Organisationsforschung) wäre allerdings ein Sachindex dringend angeraten gewesen, der dem Leser einen schnellen und je nach Interessenlage differenzierten Zugriff auf einzelne Schwerpunkte ermöglichen würde.

Anna M. Theis-Berglmair

\section{Barbara Pfetsch}

\section{Politische Kommunikationskultur}

Politische Sprecher und Journalisten in der Bundesrepublik und den USA im Vergleich Wiesbaden: Westdeutscher Verlag, 2003. 273 S.

ISBN 3-531-13708-5

Titel und Untertitel des Buches zeugen von einem ambitionierten Programm. Barbara Pfetsch (Universität Hohenheim) fokussiert auf die "Politische Kommunikationskultur“. Darunter versteht sie in der Tradition des Begriffs von „Politischer Kultur“ bei Almond und Verba die empirisch messbaren basalen Orientierungsmuster im Hinblick auf verschiedene Aspekte der politischen Kommunikation, vor allem auf Struktur, Funktion und Entwicklung öffentlicher Meinung. Diese Orientierungsmuster untersucht sie nicht gesellschaftsweit, sondern bei den Angehörigen von zwei Elitesegmenten in Deutschland und in den USA: den politischen Journalisten und den Sprechern von Regierungsorganisationen, Parlamentsfraktionen und Parteien. Sie legt der Untersuchung der politischen (Regierungs-)Kommunikationskultur also ein „Zwei-mal-zwei-De- 
sign“ zugrunde. Dabei interessiert sie besonders, ob sich Unterschiede darin zeigen, wie die Rolleninhaber ihre Interaktion sehen, deren strukturelle Bedingungen und deren Folgen für die Themenstruktur öffentlicher Meinung. Es geht also nicht um nachweisbare Effekte auf die Publikums- oder Medienagenda, sondern um die Einstellungen derjenigen, von deren Interaktion die Bildung der Medienagenda entscheidend abhängt.

Methodisch ist die Arbeit durch eine originelle Kombination aus Deuten und Messen gekennzeichnet. Auf Basis der kommunikationsund politikwissenschaftlichen Literatur kann sie analytische Hypothesen zu den Unterschieden formulieren, die dann empirisch geprüft werden. Angesichts von Umfang und Art der Datenerhebung kann man nur den Hut ziehen: Pfetsch hat Mitte der 90er Jahre 112 (!) leitfadengestützte Interviews mit politischen Sprechern und politischen Hauptstadtkorrespondenten in Washington und Bonn geführt, die sie nach dem Positionsprinzip auswählte: Sie identifizierte die für die jeweilige Regierungskommunikation wichtigsten Positionen und interviewte die Positionsinhaber. Insofern kommt die Studie einer Vollerhebung in diesen speziellen Elitensegmenten nahe. Nur gelegentlich (z. B. S. 204) wird deutlich, welche Probleme darin liegen, dass Aufschlüsse über Einstellungen in einem teil-standardisierten fremdsprachlich geführten Interview gewonnen werden sollen. Hier hätte man zumindest einige Erläuterungen $\mathrm{zu}$ Validitätsproblemen erwartet. Die Antworten wurden nach einem Kategorienschema klassifiziert und so aufbereitet, dass auch quantitative Auswertungen möglich wurden; sogar Korrelationen konnten berechnet werden, mit denen die Homogenität innerhalb der nationalen Kulturen und die $\mathrm{He}-$ terogenität zwischen den Kulturen geprüft werden. Es wird nicht ganz deutlich, in welchem Maße die Instrumente (Interviewleitfaden und Kategorienschema) während der Untersuchung verändert wurden (Hinweise darauf gibt es auf S. 119f); ohne nachträgliche Datenerhebung bzw. nachträgliche Kodierung verlören die betreffenden Häufigkeitsauszählungen ihren Sinn.

Zugespitzt zeitigt ihre Analyse folgendes Ergebnis: Wir haben es mit zwei politischen Kommunikationskulturen zu tun, deren Unterschiede sich daraus erklären, dass die Akteure der politischen Kommunikation ihre durch- aus ähnlichen Ziele in unterschiedlichen strukturellen Kontexten durchzusetzen versuchen. Die amerikanische „medienorientierte“ Kommunikationskultur folgt der Devise des „going public": Im Rahmen eines präsidialen Regierungssystems und eines weitestgehend privatwirtschaftlich organisierten Mediensystems entwickeln die Sprecher und Journalisten eine marketingorientierten Denkweise, in der die Erwartungsmuster der Öffentlichkeit bzw. des Publikums eine entscheidende politische Rolle spielen. Medienkommunikation ist integraler Bestandteil des Entscheidungsprozesses, die öffentliche Meinung ein zentraler Machtfaktor der Regierung auch für die Verhandlungen mit anderen politischen Akteuren.

Die deutsche „(partei)politische“ Kommunikationskultur folgt der Devise der „symbolischen Legitimation" bereits ausgehandelter oder zumindest vor-verhandelter Entscheidungen: Das parlamentarische Regierungssystem und ein heterogenes Mediensystem bringen Sprecher und Journalisten zwar dazu, Politik mediengerecht zu verpacken („,selling“), um öffentliche Unterstützung zu gewinnen bzw. um sich vom politischen Gegner abzusetzen, aber die Erwartungsmuster des Publikums wirken nicht konstitutiv auf Auswahl von politischen Problemen und deren Lösungen. Mediale Kommunikation setzt in der Folge von Entscheidungen ein, nicht in ihrem Vorfeld. Gerade der Kontrast zu den amerikanischen Verhältnissen macht deutlich, wie elitenzentriert die deutsche politische (Medien-)Kommunikation nach wie vor ist - in der Presse und im öffentlich-rechtlichen Rundfunk. Dies änderte sich erst durch das Hinzutreten des privaten Fernsehens.

Dieses Profil der beiden Kulturen setzt sich aus verschiedenen Elementen zusammen - wie Pfetsch detailliert zeigt: welche normativen Orientierungen jeweils als maßgebend angesehen werden, welcher Stellenwert der öffentlichen Meinung (und ihren verschiedenen Ausdrucksformen) zugeschrieben wird, welches strategische Repertoire eingesetzt wird, um die Medienagenda zu beeinflussen (z. B. über Leitmedien). Innerhalb des Rahmens der vier Gruppenaggregate werden keine weiteren Unterscheidungen mehr gemacht. Hier dürften die Daten weitere Differenzierungen erlauben z. B. müssten Subkulturen erkennbar werden oder auch Extremfälle zu identifizieren sein, die sich den gängigen Mustern entziehen.

Fazit: Die Arbeit wirft Licht in das Dunkel, 
das die Verbindungen zwischen politischer Öffentlichkeitsarbeit und Journalismus umgibt und das die Mythen gedeihen lässt. In dem Licht tritt eines klar hervor: Wenn gemeinhin von "Medien und Politik" die Rede ist, wird stillschweigend über die Bindung an die jeweiligen nationalen Verhältnisse hinweggegangen und eine universale Konstellation von „Systemen" konstruiert. Pfetsch macht deutlich, dass dies der Komplexität des Zusammenhangs nicht gerecht wird. Ein weiteres Moment wird durch die Lektüre klar: Sicherlich hat sich seit dem Zeitpunkt ihrer Datenerhebung in der politischen Kommunikation (Stichwort: „Berliner Republik“) und in der Forschung („Fra- ming") einiges verändert, und das Design der Studie verbietet die Formulierung von Aussagen über die vergangene und zukünftige Entwicklung der Kommunikationskulturen. Aber dadurch, dass Pfetsch klar die strukturellen Unterschiede zwischen den Kommunikationskulturen herausarbeitet, entkräftet sie die These, dass sich die Varianz zwischen den Nationen durch eine einseitige Konvergenz auflösen könnte („Amerikanisierung“). Damit zeigt sie, welchen enormen Erkenntnisgewinn ein komparatives Vorgehen zeitigt. Aus diesem Grunde setzt die Arbeit Maßstäbe.

Gerhard Vowe 